\title{
Germinación y crecimiento inicial de dos especies de lulo Solanum sessiliflorum Dunal o topiro y Solanum pseudolulo Heiser en condiciones naturales del corregimiento de La Troje, Chocó
}

\section{Germination and early growth of two species of lulo Solanum sessiliflorum Dunal or topiro and Solanum Heiser pseudolulo Corregimiento natural conditions of Troje, Chocó}

\author{
Haidyn Luis Moreno Mosquera*
}

\section{Resumen}

Las especies del género Solanum, representan un gran potencial productivo y socioeconómico en el departamento del Chocó, Colombia. Sin embargo, es escasa su información, siendo el propósito de esta investigación, evaluar la germinación y crecimiento inicial de dos especies de lulo (Solanum sessiliflorum o topiro y Solanum pseudolulo Heiser). Para ello, se implementó un diseño en cuadrado latino donde se ensayaron tres tratamientos con el mismo número de repeticiones T1 arena (testigo), T2 gallinaza + arena proporción (2-1) y T3 tierra de hormiga + arena proporción (2-1). En el experimento no se encontraron diferencia estadísticamente significativa en relación con el porcentaje de germinación entre las especies $(p>0,44)$ y los tratamientos $(p>0,061)$. El tiempo promedio de germinación varió de 6 a 12 días. El crecimiento inicial de las plántulas reportó altura similar de los individuos para las dos especies; sin embargo, se observó mayor altura en T3 y T2 con 16 y 17 $\mathrm{cm}$ respectivamente, a diferencia de $T 1$ en el que se obtuvo el menor crecimiento $9 \mathrm{~cm}$. En el aspecto fitosanitario, se reportó $11 \%$ de plántulas afectadas con grado de incidencia entre leve y moderado causada por larvas del género Spodoctera. Se concluyó que si bien las semillas de las dos especies evaluadas pueden germinar bien en los tres sustratos, el desarrollo de la nueva plántula es más rápido en los tratamientos enriquecidos con nutrientes (T2 y T3) en los que las plantas lograron mayor altura y número de hojas.

Palabras clave: Chocó, Crecimiento inicial Solanum, Colombia, Porcentaje germinación, Vigor germinativo.
* Investigador Asociado tipo I, Instituto de Investigaciones Ambientales del Pacífico (IIAP), Quibdó, Chocó, Colombia. e-mail:hmoreno@,iiap.org.co 


\section{Abstract}

Species of the genus Solanum, represent a great productive potential and economic partner in the department of Choco, Colombia. However there is little information about these, being the purpose of this research, evaluate germination and early growth of two species of lulo (Solanum sessiliflorum or topiro and Solanum pseudolulo Heiser). To this end, it implemented a Latin square design where three treatments with the same number of repetitions sand T1 (control), T2 chicken manure + sand ratio (2-1) and $T 3$ earth ant + sand ratio (2-1) were tested. In the experiment no statistically significant difference was found in relation to the percentage of germination among species $(p>0.44)$ and treatments $(p>0.061)$. The average germination time varied from 6 to 12 days. The initial growth of seedlings reported similar height of individuals for the two species however greater height was observed in T3 and $T 2$ with 16 and $17 \mathrm{~cm}$ respectively, unlike $T 1$ in which the lowest growth was obtained $9 \mathrm{~cm}$. In the phytosanitary aspect, $11 \%$ affected seedling grademild to moderate incidence caused by larvae of the genus Spodoctera reported. In conclusion, we see that although the seeds of the two species examined may germinate well in the three substrates, the development of new seedling is faster in treatments enriched with nutrients (T2 and T3) in which the plants achieved greater height and number of sheets.

Keywords: Chocó, Colombia, Germination percentage, Germination vigor, Initial growth Solanum.

\section{Introducción}

Solanum sessiliofurum Dunal, conocido comúnmente como cocona o topiro, es una especie nativa de América tropical distribuida alrededor de la cuenca amazónica de Brasil, Colombia,
Perú, Ecuador y Venezuela (Pereira Da Silva et al., 2011). La cocona se considera una fruta exótica y nutritiva, muy variable en cuanto a forma, tamaño, peso y composición nutricional. (Agudelo et al., 2012).

La fruta tiene importancia económica porque posee un excelente potencial a nivel industrial para la elaboración de néctar, mermeladas, helados, postres, jugos y dulces. Es una planta cuya domesticación con la incorporación de genes de especies relacionadas con el lulo apenas comienza en Colombia, con resultados promisorios y brindando así otras alternativas, a los cultivadores de lulo (ICA, 1988).

Sibien, Colombia presenta una serie de factores que favorecen y potencian la producción de este frutal, entre las que destacan: amplia variabilidad genética del taxón y especies relacionadas; nichos ecológicos apropiados para su siembra y cultivo; aceptación de los frutos por los consumidores; potencial agroindustrial; y alternativa para la reconversión productiva (Lobo-Arias, 2007), no es un país autosuficiente en su producción, razón por la cual se importa de Ecuador cerca de $20 \%$ de lo que consume (Gómez-Merino et al., 2014).

En el departamento del Chocó, Colombia, se han identificado dos especies de lulo nativo que podrían convertirse en una alternativa importante para los productores locales, por las características nutricionales y su adaptabilidad a las condiciones agroclimáticas de la zona; a pesar de esto, la información secundaria sobre las especies es limitada e insuficiente.

Tradicionalmente el Solanum sessiliflorum o topiro es cultivado en huertos caseros bajo criterios del productor local, es decir, sin prácticas agronómicas que permitan optimizar su rendimiento productivo. Por otra parte, la planta del Solanum pseudolulo Heiser (lulito de espinas) prolifera como una maleza y es generalmente considerada como especie invasora en los alrededores de las casas y zonas rural, situación que pone en peligro inminente las especies porque el desconocimiento 


\section{Bioetnia Volumen 12, 2015}

de las condiciones generales de propagación y manejo, genera limitantes para su conservación.

La semillaes el principal órganoreproductivode la gran mayoría de las plantas superiores terrestres y acuáticas. Esta desempeña una función fundamentalenlarenovación, persistencia y dispersión de las poblaciones de plantas, regeneración de los bosques y sucesión ecológica (Doria, 2010)

El estudio de la biología y fisiología de las semillas es de vital importancia para el hombre, porque la mayoría de las especies cultivadas son propagadas a partir de semillas sexuales (Russo et al., 2010). La formación, dispersión y germinación de semillas, son eventos fundamentales en el ciclo de vida de las plantas gimnospermas y angiospermas. La propagación sexual de las plantas se da por medio de las semillas, las cuales tienen la función de multiplicar y perpetuar la especie (Bradford y Nonogaky, 2007).

Debido a que existe poca información sobre la germinación de estas especies y su posible domesticación, siendo este un recurso alimenticio y económico para los pobladores de la región, es necesario implementar alternativas que garanticen el recurso para las nuevas generaciones y que a su vez permitan potenciar el aprovechamiento sostenible de las mismas. Es por ello que la presente investigación tiene como objeto evaluar las condiciones de germinación en diferentes sustratos de las semillas de dos especies de lulo en condiciones de vivero, con el fin de aporta elementos para su manejo técnico además de generar información para la toma de decisión frente a la necesidad de adelantar estrategias de producción y conservación de estas especies en el Chocó Biogeográfico.

Área de estudio. El corregimiento de La Troje, pertenece al municipio de Quibdó, Colombia, se encuentra ubicado geográficamente a $5^{\circ}$ $43^{\prime} 32,08^{\prime \prime}$ de latitud norte y a $76^{\circ} 35^{\prime} 47,6^{\prime \prime}$ de longitud oeste. Se ubica a $61 \mathrm{msnm}$; presenta una humedad relativa de $86 \%$, temperatura promedio de $25^{\circ} \mathrm{C}$, y una precipitación que oscila entre 4000-8000 mm anuales (CODECHOCÓ, 1999). Corresponde a las formaciones de zonas de vida, bosque pluvial tropical (bp-T) y bosque húmedo tropical (bh-T)(Espinal, 1977). Limita al norte con el corregimiento de Tutunendo y San Francisco de Ichó, al sur con la cabecera municipal de Quibdó, al oriente con el corregimiento de Guayabal y el río Atrato, al occidente con el río Cabí y el corregimiento de Guadalupe (Figura 1). Dentro de la vegetación del corregimiento de La Troje, se encuentran árboles de finas maderas que tienen aplicación en la industria y alimentación, además presenta unas condiciones físicas y ambientales considerables para albergar una alta riqueza de especies vegetales, en especial especies arbóreas (Forero y Gentry, 1989; Rangel et al., 2004).

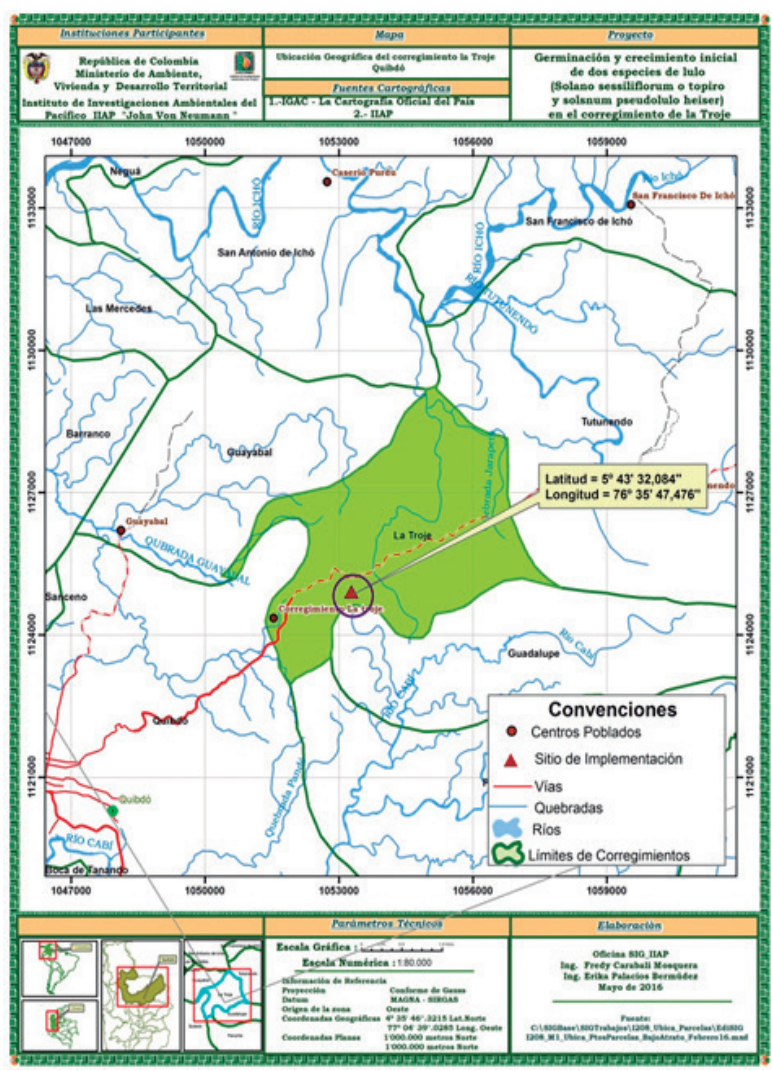

Figura 1. Área de estudio del departamento del Chocó, municipio de Quibdó, corregimiento de La Troje. 


\section{Método}

Para la obtención de semilla utilizada en los tratamientos, se seleccionaron frutos con características fenotípicas que correspondieron a 500 gramos de peso promedio, madurez fisiológica y buen estado fitosanitario (ausencia de magulladuras, heridas y/o manchas); luego se realizó un corte trasversal del fruto para extraer las semillas, que se sometieron a un proceso de limpieza para eliminar los residuos de la pulpa adherido y se secaron a temperatura ambiente durante tres días. El tiempo transcurrido entre la extracción de las semillas y el secado fue de cinco días.

El montaje de los ensayos se hizo mediante un diseño en cuadrado latino, en el que ensayaron tres tratamientos con el mismo número de repeticiones. Tratamiento (T1) o testigo, elaborado con arena; tratamiento (T2) a base de gallinaza y arena en proporción 2:1; tratamiento (T3), elaborado con tierra de hormiga y arena en proporción $2: 1$. En total se utilizaron 1800 semillas por especie y 200 semillas por repetición (Figura 2).

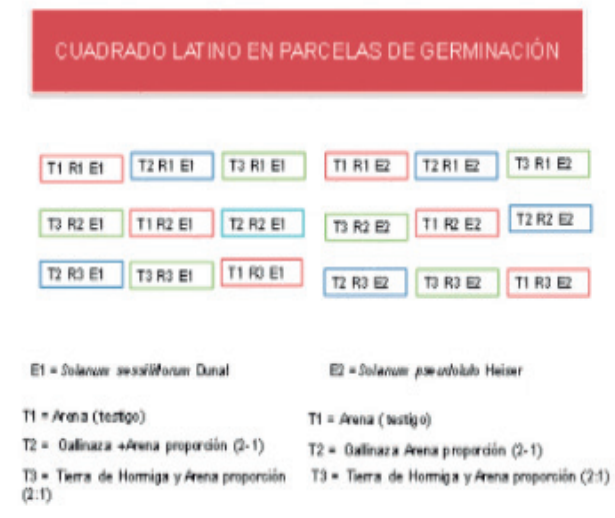

Figura 2. Diseño en cuadrado latino de los tres tratamientos seleccionados para la realización del experimento germinativo de las especies evaluadas.

Las pruebas de germinación se realizaron en eras de $750 \mathrm{~cm}^{2}(15 \times 50 \mathrm{~cm})$, cubiertas con una malla polisombra durante todo el proceso de germinación. Los datos de germinación se registraron diariamente, desde el inicio del experimento hasta su finalización, momento que se determinó cuando no aparecieron nuevas germinaciones en un período de cinco días.

En el tratamiento de los datos se calculó el porcentaje de germinación (PG), definido como el porcentaje final de semillas que germinan $(\mathrm{Sg})$ en relación con el número total de semillas sembradas $(\mathrm{Ss}) . \mathrm{PG}=(\mathrm{Sg} / \mathrm{Ss}) \times 100 \%$. Además, se registró el día de inicio de la germinación (IG), corresponde al tiempo transcurrido desde la siembra de las semillas hasta la germinación del $5 \%$ de las semillas sembradas. También se calculó elvalor germinativo(VG)(Czabator, 1962), elcual refleja los cambios en el pico de germinación, la germinación total y la velocidad de germinación de las semillas. $\mathrm{VG}=\mathrm{GDM}$ (final) $\times \mathrm{VM}$, donde VM corresponde al valor máximo o pico que se presenta entre los valores producto de la división del porcentaje acumulado de germinación y la cantidad de días que se tardó en obtenerse; GDM es la germinación media diaria, calculada como la relación entre el porcentaje final de germinación (PG) y el número de días transcurridos hasta llegar a ese valor. Este índice se expresa sin unidades.

Pasados 10 días después de la germinación, se midió la altura de 20 plántulas por replicación, para determinar el efecto del sustrato y la condición lumínica sobre el desarrollo de la plántula. Finalmente, se determinó la afección fitosanitaria de las plántulas por tratamiento, mediante la fórmula matemática $\mathrm{NPA}=\mathrm{N}$ de plantas afectadas $/ \mathrm{N}$ de plantas germinadas (NG) x 100; una vez calculado se determinó el grado de incidencia en las plantas germinadas, para lo cual se manejaron los rangos contenidos en la Tabla 1. El análisis estadístico de los datos obtenidos fue realizado a partir del programa infoStat versión 2008.

\section{Resultados y discusión}

Porcentaje de germinación. Las especies evaluadas (S. sessilifrorum o topiro y S. pseudolulo Heiser) reportaron porcentaje de germinación 
Tabla 1. Rangos y porcentajes de grado de incidencia

\begin{tabular}{lll}
\hline Clase & Descripción & Rango \\
\hline Grado 1 & Excepcional & $<1$ \\
Grado 2 & Leve & $1-20$ \\
Grado 3 & Moderado & $21-50$ \\
Grado 4 & Elevado & $>50$ \\
\hline
\end{tabular}

Tabla 2. Porcentaje de germinación por especie y por tratamiento

\begin{tabular}{lcccc}
\hline Especie & No semillas & \multicolumn{3}{c}{ Porcentaje } \\
\cline { 3 - 5 } & sembradas & T1 & T2 & T3 \\
\hline S. sessiliflorum & 200 & 86 & 89 & 90 \\
S. pseudolulo & 200 & 87 & 90 & 91 \\
\hline
\end{tabular}

superior al 85\% (Tabla 2); sin embargo, no se presentó diferencia estadísticamente significativa ( $>0.44$ ) entre estas; el valor puede estar estrechamente relacionados con las reservas energéticas de grasas, carbohidratos y proteínas que contienen las semillas, características que en el presente estudio se pudieron conservar al elegir frutos en buen estado y a la rapidez del proceso de siembra. Los resultados son superior a los registrados para $S$. sessiliflorum por Argüello (2002), quien obtuvo $63 \%$ de semillas germinadas en bandejas que contenían promix como medio de cultivo. Al igual que los reportados por Santacruz (2004) para la misma especie en Honduras (64.3\%). En cuanto a los tratamientos tan poco se presentó diferencia significativa ( $p>0,061)$; a pesar de ello los enriquecidos con materia orgánica presentaron los mayores porcentajes de germinación, siendo el T3 el que arrojó los mayores porcentajes de $90 \%$ y $91 \%$ para $S$. sessiliflorum y $S$. pseudolulo respectivamente (Tabla 2), comportamiento que concuerda con lo observado por Gómez-Merino et al. (2013) quienes afirman que los sustratos orgánicos estimulan la germinación de Solanum quitoense.
Valor máximo de germinación. Las especies en estudio iniciaron la germinación seis días después del sembrado de las semillas, proceso que se prolongó de manera periódica hasta el día 12, tiempo en que se observó la última germinación (Figura 3). La información obtenida se relaciona probablemente con la similitud en el grosor y naturaleza de la testa de las semillas, pues las especies con testa delgada y blanda presentan mayor permeabilidad y escasa dormición.

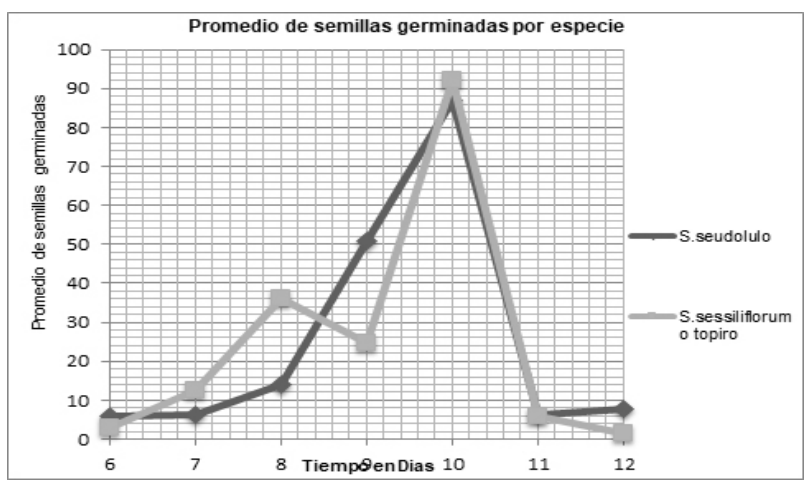

Figura 3. Promedio de semillas germinadas para S. sessiliflorum o topiro y S. pseudolulo Heiser.

Para S. sessiliflorum o topiro, se observa un aumento importante de la curva de germinación de las semillas los días 8 y 10 siendo este último quien reporta el mayor promedio de semillas germinadas (92,2); S. pseudolulo heiser mostró la misma tendencia; sin embargo, los días que reportaran mayor semillas germinadas fueron el 9 y 10 con promedios de 51 y 87 respectivamente. Los resultados obtenidos en este estudio, son consecuente con Arguello (2002), quien reporta rangos de germinación de las semillas de $S$. sessiliflorum entre 13 y 16 días una vez iniciado el proceso de germinación. Santacruz (2004), registró para la misma especie en Honduras un tiempo de germinación entre 10 y 12 días. Comparando los resultados actuales con los obtenidos con otras especies del género se evidencian variaciones en los tiempos de germinación, pudiendo ser similar en el caso de Solanum topiro con rangos entre 5 y 13 días (Bejarano y Chaverra, 2003), o menor 
al compararlos con los resultados obtenidos por Quinchia y Cabrera (2006), quienes registran para $S$. quitoense un tiempo de germinación que oscila entre 15 y 20 días después de la siembra.

La germinación media diaria (GDM) fue de $12 \%$ para el $\mathrm{T} 1$ y de $13 \%$ para $\mathrm{T} 2$ y $\mathrm{T} 3$ para las dos especies estudiadas. En la Figura 4 se observan tendencias similares de germinación entre los tratamientos para S. sessiliflorum, siendo el día 10 el que mostró el mayor número de semillas germinadas en todos los tratamientos, aunque cabe destacar el aumento en el número de las semillas presentes en el T2 (120), en relación con el T3 (89) y el T1 (68). La germinación entre los tratamientos también mostró tendencias similares en $S$. pseudolulo, exhibiendo los mayores porcentajes de germinación a los diez días. No obstante en este caso fueron los T3 (98) y T1 (93), los que presentaron los mayores cantidad de semillas germinadas, siendo superior al T2 en donde la germinación fue de 70 .

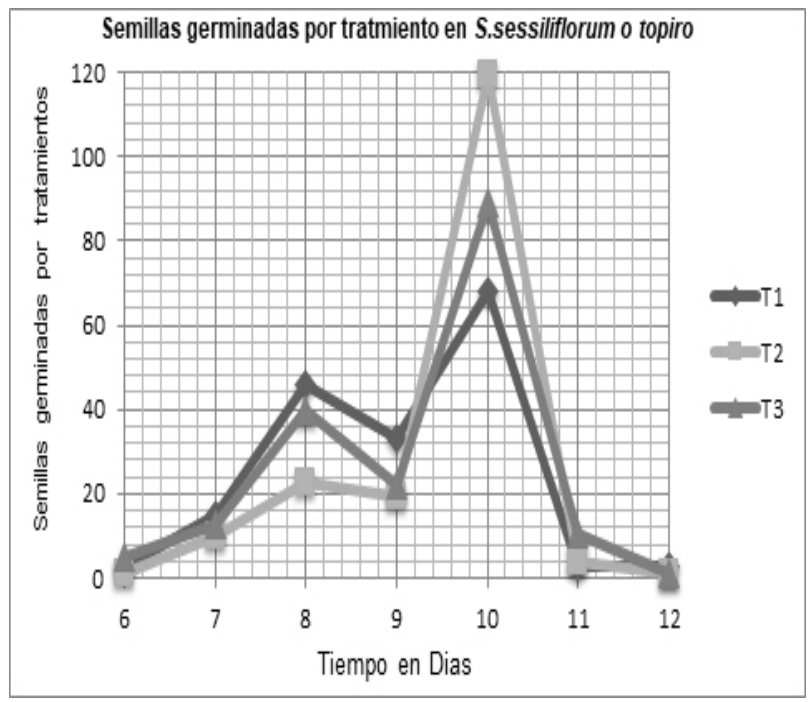

Figura 4. Semillas germinadas por tratamiento en S. sessiliflorum o topiro

El comportamiento germinativo de las especies, refleja un largo grado de senescencia (entre el día 0 y el 6), seguido por el inicio de la germinación, la cual mostró leve variación en relación con las especies, tal como lo sugiere Primack (1987) que podrían estar relacionadas con la fisiología, tamaño de las semillas y morfología de las plantas, lo que permite que cada especie se especialice en unos requerimientos de germinación reflejo de su adaptación a un ambiente determinado, hipótesis que apoya la variación en el porcentaje de germinación por tratamiento obtenida en los experimentos de esta investigación.

Crecimiento inicial de plántulas. La altura de la plántula presentó variación entre los tratamientos con compuestos orgánicos y el control, siendo mayor en los primeros con alturas entre 16 y $17 \mathrm{~cm}$ (Figura 5). Asimismo, se observó una tendencia similar en relación con el número de hojas en las que los tratamientos T2 y T3 fueron superiores (Figuras 6 y 7).

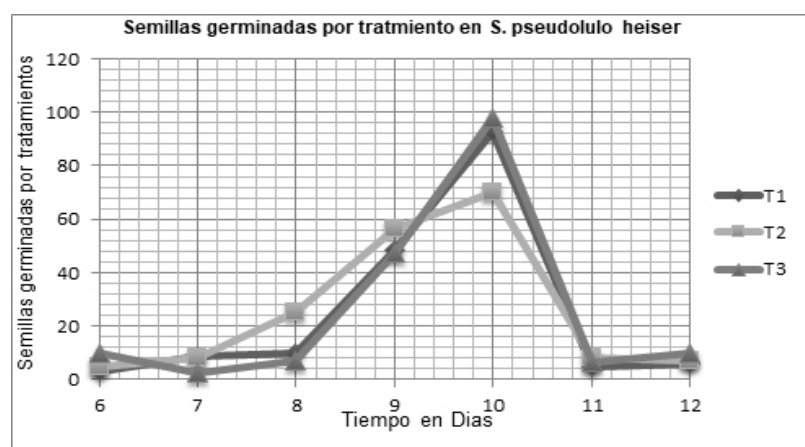

Figura 5. Semillas germinadas por tratamiento en S. sessiliflorum pseudolulo Heiser.
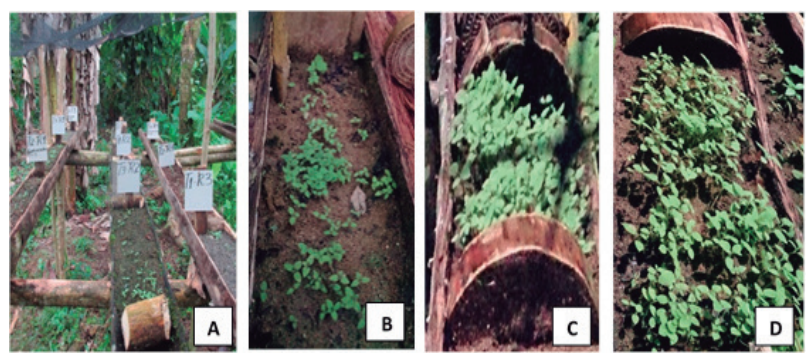

Figura 6. Germinación por tratamiento de las dos especies en estudio. A. Proceso de germinación por tratamiento B. Germinación

en T1 (arena). C. T2 (gallinaza-arena 2:1)

D. T3 (tierra de hormiga-arena 2:1).

Sin embargo, las diferencias no son evidentes entre las especies estudiadas, pues en todos los tratamientos la respuesta de las variables fue similar. Las diferencias observadas entre los tratamientos son atribuible a las características físicoquími- 
Bioetnia Volumen 12, 2015

Tabla 3. Semillas germinadas vs. porcentaje de afectación por especie evaluadas

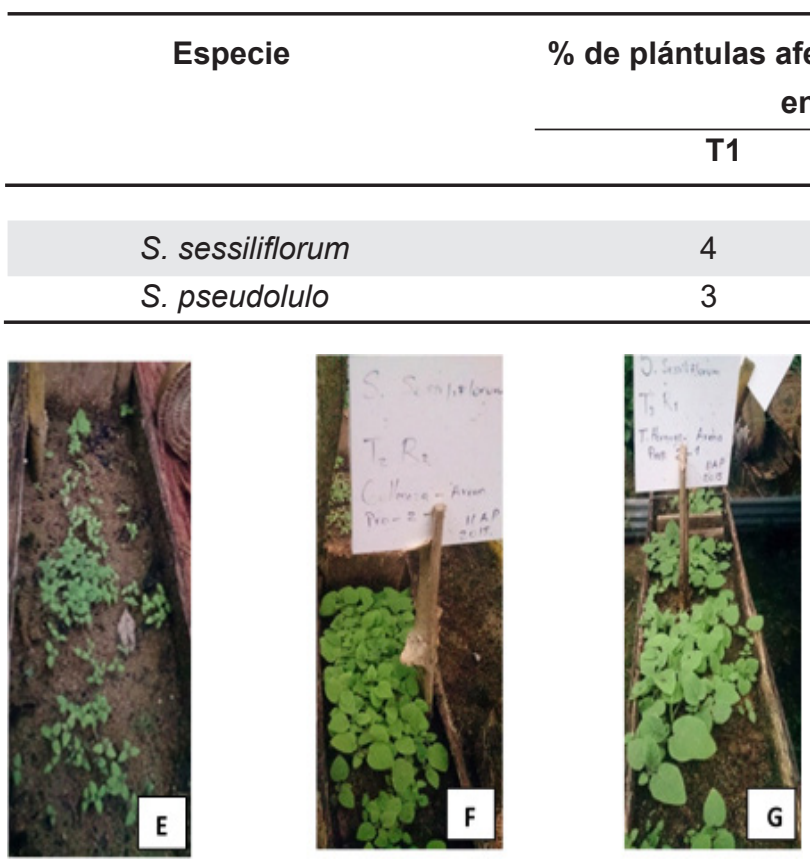

Figura 7. Altura y número de hojas lograda por las plántulas en los diferentes sustratos evaluados. E. Crecimiento en T1. F. Crecimiento T2. G. Crecimiento T3.

cas de los sustratos orgánicos como medios de desarrollo de las nuevas plantas, porque tanto la gallinaza (T2) como la tierra de hormiga (T3) presentan propiedades físico-químicas (textura y contenido nutricional) que garantizan un mejor desarrollo de la planta. Al respecto, Singh y Sainju (1998), aseguran que el crecimiento de las plantas en altura y diámetro es dependiente del aporte de agua, nutrientes, energía y aire, que un medio pueda aportarle. Lo anterior también favorece a las hojas, porque como órganos sintetizadores de carbohidratos, deben tener un buen sustrato que le garantice un suministro adecuado de nutrientes para la planta (Izco, 1997; Marschner, 1995), viéndose favorecido con el enriquecimiento orgánico con el que contaban los tratamientos T2 y T3. Al respecto, Gómez-Merino et al. (2013), afirman que las dosis bajas de composta (10\% y $20 \%)$ en combinación con dosis altas de vermicomposta, incrementan significativamente la biomasa aérea y subterránea en plantas de $S$. quitoense y mejoran sus variables morfológicas.

Presencia de plagas. La única plaga registrada en los tratamientos correspondió al género Spodoctera, familia Noctuidae, orden Lepidóptera. Los individuos de ambas especies fueron afectados con la misma intensidad, lo que permite deducir que la presencia de espinas en $S$. pseudolulo no es factor determinante en relación con la presencia de plagas; también se observó que existe una relación directamente proporcional entre el buen desarrollo de plantas y la incidencia de plagas en las mismas. Las plántulas que crecieron en los T3y T2 fueron las que presentaran mayor porcentaje de afectación para las dos especies (11\%), contrarioal T1 en el que se reportó la menor incidencia (4\%) (Tabla 3, Figura 8). Esta situación deberá propiciar alertas para el monitoreo permanente en las eras

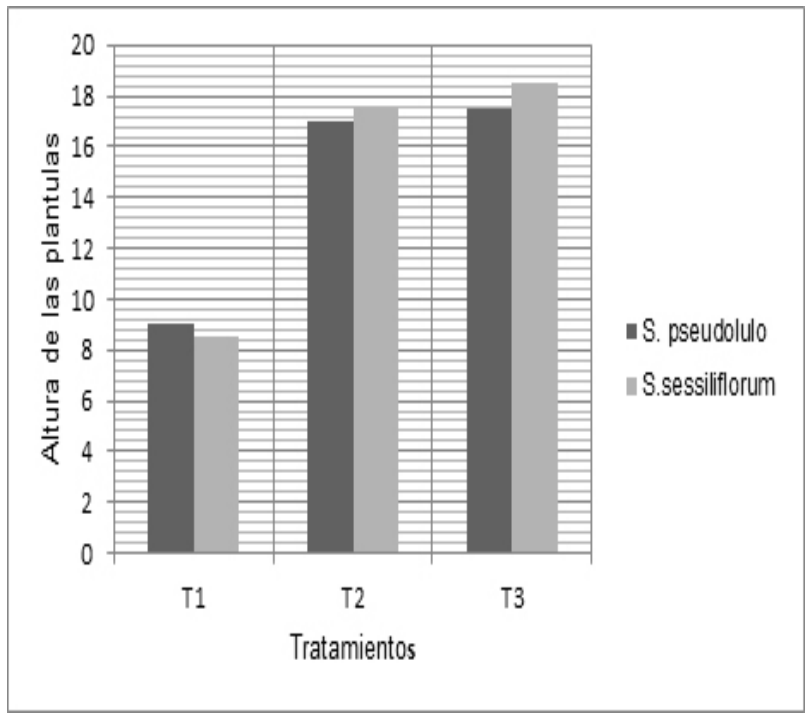

Figura 8. Incidencia de plagas defoliadoras en los tratamientos evaluados 
de germinación, trasplante a bolsas y crecimiento previo antes de la siembra en sitio definitivo, porque es evidente la presencia de defoliadores que podrían afectar el proceso de producción de material vegetal, retrasar los ciclos productivos y aumentar costos en iniciativas productivas que involucren estas especies.

Con respecto al grado de incidencia por tratamiento, las plantas presentaron mayor afectación foliar en el T3 del total sembradas: 32 son afectados de forma moderada y 15 sufrieron afectación leve, datos que son similares a los reportados en el T2: 30 y 15 respectivamente; contrario a los anteriores el $\mathrm{T} 1$ que reportó menor incidencia en las dos categorías ( 7 y 9). La afectación leve es relativamente baja, pero es importante resaltarque en este grado de incidencia se deben prender las alarmas para el control eficaz de las plagas que pueden dejar en peligro la calidad y cantidad de plántulas producidas en el vivero.

\section{Conclusión y recomendaciones}

La poca variación en el comportamiento germinativo de las semillas por especies y tratamiento, sugiere el peso de factores intrínsecos de la semilla y aspectos climáticos relacionados con la temperatura y la humedad. Se recomienda que para la obtención de las semillas se tenga en cuenta el peso del fruto (450 g) S. sessiliflorum y $(20$ g) $S$. pseudolulo, estado morfológicos y madurez fisiológica. Además, se recomiendo que el secado previo a la siembra sea a la sombra durante un tiempo aproximado de cinco días lo que disminuye el tiempo de germinación y aumenta su viabilidad.

La mezcla de sustratos de origen orgánico con arena tiene influencia positiva después de la emergencia de las plántulas acelerando su desarrollo, reduciendo el tiempo de trasplante a bolsas y siembra en sitio definitivo, situación benéfica en iniciativas productivas que incorporen las especies estudiadas. Para la producción de ma- terial vegetal del género Solanum se recomienda sustratos de origen orgánico mezclados con arena en proporción 2-1, estos reducen tiempo porque las semillas germinadas (plántulas) crecen más rápido y muestran mejor desarrollo.

Es muy probable la presencia de defoliadores en las primeras etapas de desarrollo de las plántulas en vivero; como mecanismos de prevención es necesario monitoreos frecuentes en las eras y realizar la eliminación manual de las larvas que ataquen las plántulas.

\section{Literatura citada}

Agudelo C, Igual M, Talens P, Martínez-Navarrete N. 2012. Aplicación de un método de secado combinado para la obtención de porciones de cocona (Solanum sessiliofurum Dunal) de alta calidad. Valencia: Grupo de Investigación e Innovación Alimentaria (CUINA), Universidad Politécnica de Valencia, Departamento de Tecnología de Alimentos. URL disponible en: https://riunet.upv.es/bitstream/handle/10251/33916/ Tesina.pdf

Argüello LF. 2002. Tratamientos para prolongar la conservación de la semilla de cocona (Solanum sessiliflorum Dunal). El Zamorano: Ciencia y producción agropecuaria; $35 \mathrm{pp}$.

Bradford KJ, Nonogaki H. 2007. Seed development, dormancy and germination. Oxford: Blackwell Publishing; p. 224-47. URL disponible en: http://onlinelibrary. wiley.com/doi/10.1002/9780470988848.fmatter/pdf

Bejarano J, Chavera F. 2003. Cultivo de lulo (Solanum topiro Humb); su manejo agronómico y de postcosecha bajo condiciones agroambientales en el municipio de Quibdó(Chocó). (Tesis de grado). Quibdó: Universidad Tecnológica.

CODECHOCÓ. 1999. Corporación Autónoma para el Desarrollo del Chocó. Plan de Desarrollo Territorial 1988-2002, Quibdó, Chocó.

Czabator FJ. 1962. Germination value: an index combining speed and completeness of pine seed germination. Forest Sci. 8(4): 386-96.

Doria J. 2010. Generalidades sobre las semillas: su producción, conservación y almacenamiento. Cultrop. 31 (1): 74-85. URL disponible en: http://scielo.sld.cu/pdf/ ctr/v31n1/ctr11110.pdf

Espinal S. 1977. Zonas de vida o formaciones vegetales de Colombia. Bogotá: Instituto Geográfico Agustín Codazzi. Volumen XIII, memorias explicativas. 


\section{Bioetnia Volumen 12, 2015}

Forero E, Gentry AH. 1989. Lista anotada de las plantas del departamento del Chocó, Colombia. Bogotá: Instituto de Ciencias Naturales, Museo de Historia Nacional; 142 pp.

Gómez-Merino FC, Trejo-Téllez LI, García-Albarado JC, Cadeña-Íñiguez J. 2014. Lulo (Solanum quitoense [Lamarck.]) como cultivo novedoso en el paisaje agroecosistémico mexicano. Rev Mex Cienc Agric Pub Esp. 9: 1741-53. URL disponible en: http://www.redalyc. org/pdf/2631/263137781017.pdf

Gómez-Merino FC, Trejo-Téllez LI, García-Albarado JC, Morales-Ramos V. 2013. Lulo (Solanum quitoense Lamarck.) como nuevo elemento del paisaje en México: germinación y crecimiento en sustratos orgánicos. Rev Mex Cienc Agric Pub Esp. 5: 877-87. URL disponible en: http://www.redalyc.org/articulo. oa? id=263128352002

Instituto Colombiano Agropecuario(ICA). 1988. Fitotoxicidad de N.O. de potreros donde se aplican hormonales, virosis lulo. Medellín: ICA; p.18

Izco J. 1997. Botánica. Madrid: McGraw-Hill/Interamericana de España, SA. Disponible en biblioteca en carta 2005 de Microsoft Corp.

Lobo-Arias M. 2000. Papel de la variabilidad genética en el desarrollo de los frutales andinos como alternativa productiva. Memorias del III Seminario de Frutales de Clima Frío Moderado. Noviembre 15 al 17, Manizales, Colombia; pp. 27-36.

Marschner H. 1995. Mineral nutrition of higher plants. $2^{\text {nd }}$ ed. London: Editorial Academic Press; 889 pp. URL disponible en: http://www.sciencedirect.com/science/ book/9780124735422

Quinchia CF, Cabrera CA. 2006. Manual técnico del cultivo del lulo (Solanum quitoense Lam.) en el departamento de Huila. Neiva: Gobernación del Huila, Secretaría de Agricultura y Minería. URL disponible en: https:// es.scribd.com/doc/62377383/Manual-Tecnico-DelLulo-en-El-Huila
Rangel JO, Rivera O, Giraldo D, Parra C, Molina JC, Gil I, et al. 2004. Catálogo de espermatofitos en el Chocó Biogeográfico. pp. 105-439. En: Rangel JO (ed). Bogotá: Colombia diversidad biótica IV: el Chocó Biogeográfico/costa pacífica. URL disponible en: https:// www.google.com.co/search?q=Cat $\% \mathrm{C} 3 \% \mathrm{~A} 1 \log 0+-$ de+espermatofitos + en + el + Choc $\% \mathrm{C} 3 \% \mathrm{~B} 3+\mathrm{Biogeo}-$ gr $\% \mathrm{C} 3 \%$ Alfico\&tbm $=$ isch \& tbo $=$ u\&source $=$ univ\&$\underline{\mathrm{sa}=X \& \mathrm{ved}=0 \text { ahUKEwivt-7Qn_XWAhXGQCYKHa- }}$ JOAYUQsAQIOA\&biw=1523\&bih=311\#imgrc $=$ rbL$\underline{\text { mxvljAqaxsM }}$

Russo RS, Zhou Y, Haase KB, Wingenter OW, Frinak EK, et al. 2010. Temporal variability, sources, and sinks of C 1-C 5 alkyl nitrates in coastal New England. Atmos Chem Phys. 10(4): 1865-83. URLdisponible en: https:// www.atmos-chem-phys.net/10/1865/2010/

Santacruz MA. 2004. Estudio fenológico y reproductivo de la naranjilla (Solanum quitoense Lam), cocona (Solanum sessiliflorum Dunal) y uchuva(Physalis peruviana Lam). Tesis para optar el título de Ingeniera Agrónoma. Honduras: El Zamorano; 67 pp. URL disponible en: https://bdigital.zamorano.edu/bitstream/11036/2029/1/ CPA-2004-T050.pdf

Singh B, Sainju U. 1998. Soil physical and morphological properties and root growth. HortScience. 33(6): 966-71.

Pereira da Silva DF, Rocha RHC, Chamhum Salomão LC. 2011. Postharvest quality of cocona (Solanum sessiliflorum Dunal) stored under ambient condition. Rev Ceres. 58(4): 476-80. URL disponible en: http://www. scielo.br/pdf/rceres/v58n4/a11v58n4.pdf

Primack RB. 1987. Relationships among flowers, fruits and seeds. Ann Rev Ecol Syst. 18: 409-30. URL disponible en: https://www.researchgate.net/publication/234150538 Relationships_Among_Flowers Fruits And Seeds 